\title{
Universal School-Based Intervention Does Not Reduce Socioeconomic Inequalities in Weight Status among Adolescents
}

\author{
Florian Manneville, MPH,' Abdou Y. Omorou, MD, PhD, , ${ }^{1,2}$ Karine Legrand, PhD, 1,2 \\ Johanne Langlois, PhD, ${ }^{1,3}$ Edith Lecomte, PhD, ${ }^{3}$ Francis Guillemin, MD, PhD, 1,2 \\ Serge Briançon, MD, PhD'; and the PRALIMAP Trial Group*
}

\section{Abstract}

Background: Differences in weight status by socioeconomic position occur during adolescence and is known as weight social gradient (WSG). The PRALIMAP (Promotion de l'ALIMentation et de l'Activité Physique) trial highlighted that a universal intervention was effective in reducing overweight and obesity prevalence among adolescents. However, its ability to reduce the WSG is unclear. This study aimed to evaluate the impact of the PRALIMAP intervention on the WSG among adolescents.

Methods: Data were obtained from 3504 adolescents who participated in the school-based PRALIMAP trial between 2006 and 2009. The PRALIMAP study consisted of three interventional strategies: educational, environmental, screening and care. Weight status was assessed by body mass index (BMI) $z$-score and overweight and obesity prevalence. The "indice de position sociale des élèves" was used to measure adolescents' socioeconomic status. Linear regression and hierarchical models were used to assess the WSG and its evolution, respectively.

Results: Among all adolescents (14-18 years old), significant WSG was identified at baseline: BMI $z$-score $(\beta=-0.06 ; p<0.0001)$, overweight and obesity prevalence $(\beta=-2.86 ; p<0.0001)$. Overall, the intervention conferred no significant reduction in the WSG: BMI $z$-score $(\beta=-0.01[-0.02$ to 0.01$], p=0.48)$, overweight and obesity prevalence $(\beta=-0.01$ [ -0.07 to 0.05$], p=0.73)$. Similar results were observed for adolescents whatever the interventional strategy they benefited from in the PRALIMAP trial.

Conclusions: This study provided no evidence that the PRALIMAP universal intervention was effective in reducing the socioeconomic inequalities in weight status among adolescents. Different interventions that account for social differences should be considered to tackle these inequalities.

Keywords: adolescents; universal intervention; weight social gradient

\section{Background}

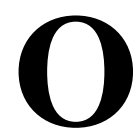
verweight and obesity among adolescents have become major global public health concerns. The worldwide prevalence of overweight and obesity has substantially increased during the past three decades. ${ }^{1}$ In France, the overweight and obesity prevalence among children and adolescents is estimated at $\sim 17 \%{ }^{2}$ Adolescents who are overweight or obese are more likely to continue to have obesity into adulthood and are at risk of several diseases such as diabetes, cardiovascular diseases, and certain forms of cancer. ${ }^{3-5}$
In high-income countries, low socioeconomic status (SES) has been identified as strongly related to overweight and obesity prevalence. ${ }^{6,7}$ The highest rates are observed among the lowest socioeconomic groups, which increases their risk of morbidity and mortality. This observation reflects socioeconomic inequalities in weight status, which concern all socioeconomic groups, not just a particularly disadvantaged socioeconomic group, and follow a weight social gradient (WSG). ${ }^{8}$ That is, overweight and obesity prevalence linearly decreases with increasing SES. ${ }^{8,9}$

Recent studies suggested that the rapid increase in overweight and obesity prevalence among adolescents has

\footnotetext{
'University of Lorraine, APEMAC, Team MICS, Nancy, France.

${ }^{2}$ CHRU-Nancy, INSERM, University of Lorraine, CIC, Clinical Epidemiology, Nancy, France.

${ }^{3}$ National Conservatory of Arts and Crafts (CNAM), Scientific and Technical Institute for Food and Nutrition (ISTNA), Nancy, France.

*For members of the PRALIMAP Trial Group, see Acknowledgment section.
} 
plateaued, but socioeconomic inequalities in weight status are persistent or still increasing during this phase of life. ${ }^{10-12}$ For example, a French study highlighted no significant evolution of overweight and obesity prevalence among adolescents from 2006 to $2015 .^{2}$ However, trends in overweight and obesity prevalence differ according to adolescents' SES and thus may widen the WSG. ${ }^{10}$ Indeed, in the 2002-2014 health behavior in school-aged children study, the authors showed that WSG among adolescents remained unchanged or have become wider since 2002 in European countries. ${ }^{11}$

There is evidence of the effectiveness of universal interventions (i.e., interventions delivered equally to the whole population) to prevent overweight and obesity among adolescents. ${ }^{13}$ In a systematic review, the authors identified six universal interventions aimed at preventing obesity among adolescents; four were effective. ${ }^{13}$ Nevertheless, given that universal interventions do not account for social differences, it is necessary to evaluate their impact on the WSG. For instance, a universal intervention may be inequitable in its impact because of inequities in uptake. ${ }^{14}$ With that in mind, Whitehead called for an evaluation of all interventions aimed at promoting health for their impact on WSG. ${ }^{15}$ Despite these recommendations, little is known about the impact of universal interventions on the WSG among adolescents. ${ }^{14,16}$ In a systematic review, the authors ${ }^{16}$ reported all existing interventions that might reduce WSG, and none were specific to adolescents.

The PRALIMAP (Promotion de l'ALIMentation et de l' Activité Physique) study was a 2-year universal schoolbased intervention aimed at preventing and reducing overweight and obesity among adolescents. ${ }^{17}$ Bonsergent et al. ${ }^{18}$ have highlighted the effectiveness of the PRALIMAP trial but the question remains about its ability to reduce the WSG. Therefore, the objective of this study was to evaluate the impact of the PRALIMAP universal intervention on the WSG among adolescents.

\section{Methods}

\section{The PRALIMAP Trial}

In this study, we used data from the PRALIMAP trial. It was a $2 \times 2 \times 2$ factorial cluster randomized controlled trial implemented in 24 state high schools in northeastern France between 2006 and 2009 (two academic years). This trial aimed to assess the effectiveness of three interventional strategies: "environmental," "educational," and "screening and care" - in preventing and reducing overweight and obesity among adolescents. The "environmental" strategy consisted of improving dietary and physical activity offerings in high schools; the "educational" strategy consisted of nutritional lessons and working groups; and the "screening and care" strategy consisted of detecting overweight or obesity and eating disorders among adolescents and proposing, if necessary, an adapted care management program of seven educational sessions.

The major finding of the PRALIMAP study was that of the three strategies implemented in the trial, the "screening and care" strategy may be an effective way to prevent and reduce overweight and obesity among adolescents. ${ }^{18}$ Each high school was assigned to receive or not the three strategies ("educational," "screening and care," and "environmental") according to a $2 \times 2 \times 2$ factorial cluster randomization. Data were collected at grades $10(\mathrm{~T} 0=$ baseline $)$, 11 (T1), and 12 (T2) entry. Every academic year, an information letter was given to parents by high schools. If parents did not want data about their children to be collected, they had to inform high schools through the school nurse by writing and signing a letter in which they indicated their refusal. Adolescents were also given written and oral information and had the right not to participate. The study protocol was previously published and fully described in detail elsewhere. ${ }^{17}$ The PRALIMAP trial was approved by the French National Commission for Computing and Liberties [CNIL] (no. 906312) and was registered at ClinicalTrials.gov (no. NCT00814554).

Among the 5354 adolescents included in the PRALIMAP trial, 3504 (65.4\%) completed the 2-year intervention and made up our study sample.

\section{Measurements}

Anthropometric data. Weight and height were measured twice in a single session by high school nurses. The body mass index (BMI) was calculated as weight/height ${ }^{2}$ from the mean of two measurements. Because BMI differs between boys and girls and is expected to increase with age during adolescence, we used an indicator that does not depend on age and sex; the BMI $z$-score. BMI $z$-score represents an age- and sex-specific BMI, according to the World Health Organization reference and allows direct comparison of BMI changes between boys and girls of different ages. ${ }^{19}$ BMI $z$-score was calculated by measuring the distance (in standard deviations) between the measured BMI and the mean BMI of a World Health Organization reference population ${ }^{19}$ and was considered as continuous variable in the statistical analysis. Age- and sex-specific cutoff values of the International Obesity Taskforce were used to define overweight and obesity. ${ }^{20}$ The BMI $z$-score was the primary outcome and overweight and obesity prevalence was the secondary outcome.

Sociodemographic data. Sociodemographic data were collected once at T0. Data on age, sex, administrative area of high school (Meurthe-et-Moselle, Moselle, Meuse and Vosges), school boarding status (nonboarder, half-boarder, or full boarder), family composition (two- or single-parent), school type (general and technological or professional), social and professional class of the family head according to the definition of the national institute of statistical and economic studies in France (executives; intermediate jobs; farmers, shopkeepers, craftsmen and managers; employees and workers; inactive [retired or unemployed]), ${ }^{21}$ and schooling placement (classical or advanced [ $\leq 15$ years] or 
late [ $>15$ years]) were obtained from the Board of Education database. Perceived family income level (low, moderate, or high) and type of residence (rural or urban) were additional sociodemographic data collected by selfadministered questionnaires completed by adolescents at $\mathrm{T} 0$.

From these data, the "indice de position sociale des élèves" (IPSE) was derived to measure adolescents' SES. Because of its multidimensional nature, SES involves the use of several sociodemographic characteristics to measure it. Moreover, adolescents' SES depends on sociodemographic characteristics of both parents. ${ }^{22}$ It is therefore necessary to use an indicator that summarizes these characteristics to measure adolescents' SES, such as the IPSE. The IPSE is a quantitative index developed by the statistical entity of the French Ministry of Education. ${ }^{23}$ The methodology used to develop IPSE is similar to that used by Ganzeboom to build the standard international socioeconomic index of occupational status. ${ }^{24}$ It consists in summarizing sociodemographic characteristics that define both parents' occupations (according to the definition of the national institute of statistical and economic studies in France ${ }^{21}$ ) into a reference value. To compute reference values, authors analyzed parents' sociodemographic characteristics (educational level, material conditions, cultural capital, and cultural practices) of 29,544 French children in grade 6 through multiple component analyses. We applied these reference values to parents' occupations available in our database for defining adolescents' SES.

Reference values range from 40 (lowest SES) to 179 (highest SES) and was rescaled from 1 (lowest SES) to 10 (highest SES) for this study. To have a sufficient number of adolescents in each SES group, the IPSE was categorized into five classes to investigate the WSG: [1-2] highly less advantaged; [3-4] less advantaged; [5-6] intermediate; [7-8] advantaged; and [9-10] highly advantaged.

\section{Statistical Analysis}

Descriptive statistics were used to report baseline IPSE classes, sociodemographic and anthropometric characteristics of included adolescents. Continuous variables are described as mean \pm standard deviation and categorical variables as number (\%).

First, we examined baseline WSG as a linear association between SES (IPSE classes) and weight status outcomes (BMI $z$-score and overweight and obesity prevalence) using unadjusted linear regression models. In these analyses, IPSE was considered as a discrete variable to obtain the $p$-value and the slope (beta) of linear trend test. The significance of the linear trend test evidenced the presence of a WSG and the slope (beta) indicated its magnitude. WSG was the slope (beta) of IPSE plotted against weight status.

Second, we analyzed the impact of the PRALIMAP universal intervention on WSG among adolescents. Changes in weight status outcomes (T2-T0) were calculated for each IPSE class by using paired Student's $t$-test for BMI $z$-score, and McNemar's test for overweight and obesity prevalence. Then we compared WSG changes between IPSE classes by hierarchical models that included within-adolescents and within-high-school variance. IPSE was declared as a discrete variable and models were adjusted for age and sex.

Third, we carried out the same analyses as previously among adolescents who received or not "screening and care," "environmental," and "educational" strategies (available in Supplementary Tables S1 and S2). Given the $2 \times 2 \times 2$ factorial design of the PRALIMAP trial (adolescents could benefit from several strategies), the effect of each strategy was adjusted for the other strategies in addition to age and sex. ${ }^{25}$

To complement the results and to contribute to their understanding, we computed Supplementary analyses for BMI (available in Supplementary Tables S3 and S4).

A value of $p<0.05$ was considered statistically significant. Statistical analyses involved use of SAS v9.4 (SAS Institute, Inc., Cary, NC).

\section{Results}

\section{Sociodemographic and Weight Status \\ Characteristics of Adolescents}

Table 1 describes the sociodemographic and weight status characteristics of the 3504 adolescents. The mean ( \pm standard deviation) age was 15.2 ( \pm 0.6 years) and 2022 $(57.7 \%)$ of the adolescents were girls. Overall, $85.1 \%$ of adolescents attended general and technological high schools, $73.2 \%$ were in classical or advanced placement at school, and $69.4 \%$ were half-boarders. Approximately half $(48.2 \%)$ lived in urban areas, $84.7 \%$ were in two-parent families, $58.6 \%$ perceived a high family income level and the family heads were predominantly employees or workers $(39.4 \%)$. IPSE classes consisted of highly less advantaged $(n=520,14.8 \%$ of adolescents), less advantaged ( $n=956$, $27.3 \%)$, intermediate $(n=1027,29.3 \%)$, advantaged $(n=$ $683,19.5 \%)$, and highly advantaged $(n=318,9.1 \%)$. The BMI $z$-score was $0.23 \pm 1.0$ and $18.2 \%$ of adolescents were overweight or obese.

\section{Baseline WSG}

Baseline unadjusted linear associations between SES (IPSE classes) and weight status outcomes (BMI $z$-score and overweight and obesity prevalence) are given in Table 2. For the entire sample, we evidenced significant baseline WSG. BMI $z$-score linearly decreased with increasing SES $(\beta=-0.06, p<0.0001)$. Overall, significant linear associations were also identified between SES and overweight and obesity prevalence $(\beta=-2.86, p<0.0001)$. Similar results were found whatever the interventional strategy adolescents benefited from (Supplementary Tables S1 and S2).

\section{Impact of Universal Intervention on WSG}

The graphical representation of age- and sex-adjusted WSG for BMI $z$-score and overweight and obesity prevalence at $\mathrm{T} 0$ and $\mathrm{T} 2$ is given in Figure 1. 


\section{Table I. Baseline Sociodemographic and Weight Status Characteristics of Adolescents $(n=3504)$}

\section{Characteristics}

Sociodemographic

Age (years), mean $\pm S D$

Sex

$$
\begin{aligned}
& \text { Boys } \\
& \text { Girls }
\end{aligned}
$$

School type

General and technological

Professional

Administrative area of high school

Meurthe-et-Moselle

Moselle

Meuse and Vosges

School placement

Classical or advanced ( $\leq 15$ years)

Late ( $>15$ years)

School boarding status

Nonboarder

Half-boarder

Full-boarder

Missing

Family composition

Two-parent

Single-parent

Missing

Residence

Rural

Urban

Missing

Perceived family income level

Low or average

High

Missing

Social and professional class of the family

Executives

Intermediate jobs

Farmers, shopkeepers, craftsmen, managers
Table I. Baseline Sociodemographic and Weight Status Characteristics

of Adolescents $(n=3504)$ continued
Number of adolescents

\begin{tabular}{l|l}
$n$ & $\%$
\end{tabular}

3504

$15.2 \pm 0.6$

1482

42.3

2022

57.7

2981

85.1

523

1090

31.1

1313

37.5

II0I

31.4

2566

73.2

938

26.8

729

20.8

2432

332

II

2968

84.7

509

1677

1690

137

1308

2054

142

694

950

253

\begin{tabular}{l|r|r}
\multirow{2}{*}{ Characteristics } & \multicolumn{2}{|c}{$\begin{array}{c}\text { Number of } \\
\text { adolescents }\end{array}$} \\
\cline { 2 - 3 } Employees and workers & \multicolumn{1}{|c}{$n$} & $\%$ \\
Inactive (retired, unemployed) & 1382 & 39.4 \\
Missing & 198 & 5.7 \\
IPSE classes & 27 & 0.8 \\
Highly less advantaged & & \\
Less advantaged & 520 & 14.8 \\
Intermediate & 956 & 27.3 \\
Advantaged & 1027 & 29.3 \\
Highly advantaged & 683 & 19.5 \\
Weight status & 318 & 9.1 \\
BMl z-score, mean \pm SD & 3504 & $0.23 \pm 1.0$ \\
Overweight and obesity prevalence & & \\
Yes & 637 & 18.2 \\
No & 2867 & 81.8 \\
\hline
\end{tabular}

Data are given as $n(\%)$ unless otherwise indicated.

BMI, body mass index; IPSE, indice de position sociale des élèves; SD, standard deviation.
Table 3 shows the evolution of WSG for BMI $z$-score from $\mathrm{T} 0$ to $\mathrm{T} 2$. For all adolescents, between $\mathrm{T} 0$ and $\mathrm{T} 2$, the BMI $z$-score significantly decreased (range, -0.07 to -0.03 ) in each IPSE class. The WSG did not significantly change during the intervention $(\beta=-0.01 ; 95 \%$ confidence interval $[-0.02$ to 0.01$], p=0.48)$. Compared with adolescents from highly advantaged class, those from other IPSE classes showed no significant change in BMI $z$-score. On subgroup analysis, similar results were observed, whether adolescents benefited from the "screening and care," "environmental," and "educational" strategies or not (Supplementary Table S1). We found a significant change in BMI $z$-score for each IPSE class between T0 and T2 without a significant reduction in WSG.

The evolution of WSG for overweight and obesity prevalence from T0 to T2 is given in Table 4. Except for adolescents from intermediate class, the prevalence of overweight and obesity did not significantly decrease from $\mathrm{T} 0$ to $\mathrm{T} 2$. After the intervention, there was no significant change in the WSG ( $\beta=-0.01[-0.07$ to 0.05$], p=0.73)$. Whatever the interventional strategy adolescents received, analogous results were found (Supplementary Table S2). We found no significant decrease in overweight and obesity prevalence for each IPSE class and no significant reduction in WSG from T0 to T2. 


\begin{tabular}{|c|c|c|}
\hline SES & $\begin{array}{l}\text { BMI z-score } \\
\text { Mean (SD) }\end{array}$ & $\begin{array}{c}\text { Overweight/ } \\
\text { obesity } \\
\text { prevalence } \\
\%\end{array}$ \\
\hline Highly less advantaged $(n=520)$ & $0.30(1.1)$ & 22.50 \\
\hline Less advantaged $(n=956)$ & $0.31(1.0)$ & 20.19 \\
\hline Intermediate $(n=1027)$ & $0.24(1.0)$ & 18.50 \\
\hline Advantaged $(n=683)$ & $0.14(1.0)$ & 15.96 \\
\hline Highly advantaged $(n=3 \mid 8)$ & $0.07(0.8)$ & 8.81 \\
\hline Test for linear trend ${ }^{a}$ & $\begin{array}{l}\beta=-0.06 \\
p<0.0001\end{array}$ & $\begin{array}{l}\beta=-2.86 \\
p<0.0001\end{array}$ \\
\hline
\end{tabular}

SES defined according to IPSE score ([1-2] highly less advantaged, [3-4] less advantaged, [5-6] intermediate, [7-8] advantaged, and [9-10] highly advantaged). Bold indicates statistical significance. aIPSE used as discrete variable. The significance $(p)$ of the linear trend test evidenced the presence of a WSG and the slope $(\beta)$ indicated its magnitude.

SES, socioeconomic status; WSG, weight social gradient.

Supplementary Figure S1 and Supplementary Tables S3 and S4 present results for BMI.

\section{Discussion}

This study highlights that the effectiveness of the PRALIMAP universal intervention to reduce overweight and obesity among adolescents in a high school setting over a 2-year period did not differ by SES. It showed a WSG among adolescents at T0 and T2: the lower the SES, the higher the BMI $z$-score and overweight and obesity prevalence. Adolescents from higher socioeconomic groups were more likely to have lower BMI $z$-score and overweight and obesity prevalence than adolescents from lower socioeconomic groups. However, the PRALIMAP universal intervention did not significantly reduce the WSG among adolescents.

This study identified a baseline WSG among adolescents. Ours results are consistent with the literature. ${ }^{26,27}$ Goodman et al. ${ }^{26}$ highlighted a highly significant linear increase in relative risk of adolescent obesity with SES. In a systematic review from 1990 to 2013, the authors reported evidence of associations between SES and adolescent weight status. ${ }^{27}$ They observed an inverse relation between weight status and SES for children or adolescents in $60.4 \%$ of the 158 included studies. In addition, this proportion increased to $66 \%$ for studies that used International Obesity Taskforce cutoff values.

Unlike for BMI $z$-score, we did not find a significant decrease in prevalence of overweight and obesity in each socioeconomic group during the intervention. This observation may be because of a lack of statistical power. Indeed, we found a significant decrease in prevalence of overweight and obesity only among adolescents from intermediate socioeconomic group, the most represented in our study. In addition, results of the PRALIMAP trial showed a significant decrease of $1.38 \%$ in overweight and obesity prevalence among all adolescents. ${ }^{18}$ However, the PRALIMAP study was not originally powered to show a decrease in overweight and obesity prevalence from $\mathrm{T} 0$ to $\mathrm{T} 2$ in each socioeconomic group. ${ }^{17}$

During the intervention, no significant changes occurred in the WSG. This finding is consistent with results from a systematic review by Moore et al. ${ }^{14}$ The authors stressed that, in most universal school-based interventions, socioeconomic inequalities are rarely considered part of the problem to address. Thus, the assessment of the impact of these interventions on socioeconomic inequalities in health often does not reach significance, which may be the case in our study because the PRALIMAP trial was not originally powered to detect a differential impact of the intervention between socioeconomic groups. Indeed, from a sample size of 3504 adolescents, an alpha risk of 5\% and a power of $80 \%$, we would have evidenced a reduction of 0.023 in WSG from T0 to T2 for BMI $z$-score. Our findings are also congruent with Bambra et al. ${ }^{16}$ who found no significant WSG changes in a meta-analysis of 11 universal interventions that might reduce existing inequalities in the prevalence of obesity among young people. Another explanation of our result could be that the PRALIMAP universal intervention was equally uptaken by adolescents, whatever their SES. As a result, there was neither a reduction nor an increase in the WSG. In their systematic review, Hillier-Brown et al. ${ }^{28}$ concluded that universal interventions have the potential to slow the widening of the WSG. The authors identified a 4-year school-based multicomponent education and exercise universal intervention conducted in France and aimed at preventing overweight among adolescents. ${ }^{29}$ At 4 years, adolescents who received the intervention exhibited a lower increase in BMI $(p=0.01)$ and age- and sex-adjusted BMI $(p<0.02)$ than adolescents in the control group, with no differential effects by SES.

On subgroup analyses, we did not evidence the effectiveness of the "screening and care," "educational," and "environmental" strategy to reduce the WSG (Supplementary Tables S1, S2, S4). Thus, the lack of evidence of effectiveness of such strategies to reduce the WSG may not be related to their design but rather to the universal principle of the PRALIMAP intervention itself. These different points led us to suggest that social differences should be accounted for in interventions aimed at addressing socioeconomic inequalities in weight status. Specifically and according to Marmot ${ }^{30}$ interventions should be universal and proportionate (i.e., with a scale and intensity that is proportionate of the level of disadvantage). For example, Legrand et al. ${ }^{31}$ conducted a universal and proportionate 

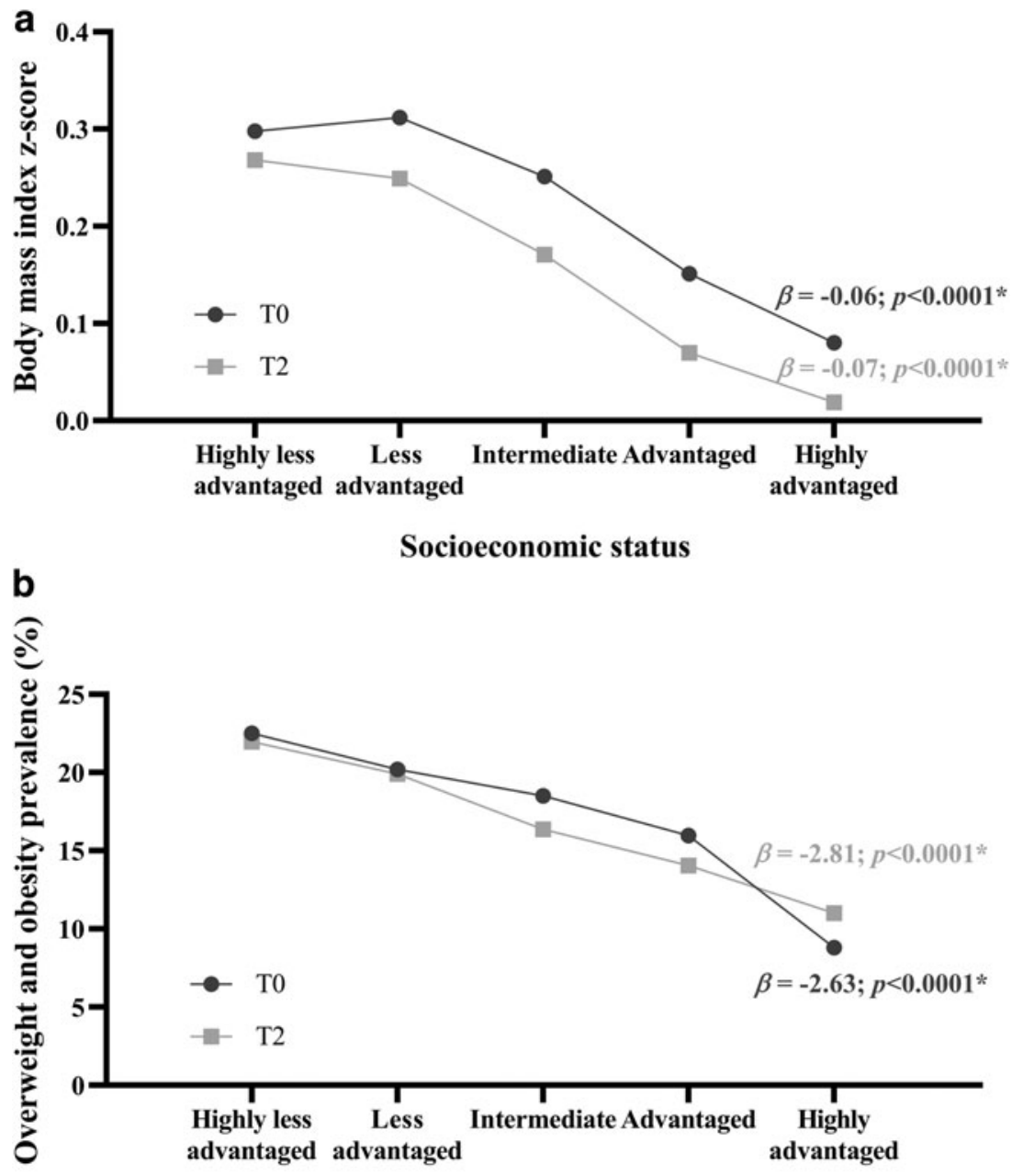

Socioeconomic status

Figure 1. Weight social gradient for (a) body mass index $z$-score and (b) overweight and obesity prevalence at baseline (T0) and end of the intervention (T2) for the whole sample. SES was defined according to IPSE value ([1-2] highly less advantaged, [3-4] less advantaged, [5-6] intermediate, [7-8] advantaged, and [9-10] highly advantaged). T0, inclusion; T2, end of intervention. *Regression coefficient and corresponding $p$-value of age- and sex-adjusted linear trend test between SES and weight status outcomes at T0 and T2. IPSE, indice de position sociale des élèves; SES, socioeconomic status.

intervention to reduce WSG among adolescents. Specifically, a standard-care management was proposed for all adolescents, whereas a strengthened-care management was proposed for only adolescents from low socioeconomic group. The standard-care management included five 2-hour group sessions on physical activity and diet, and the strengthened-care management consisted of adapted activities for overcoming barriers that are supposed to contribute to WSG.

\section{Study Limitations and Strengths}

This study has some limitations. First, the PRALIMAP trial was not originally powered to analyze the impact of the intervention on WSG. However, this study followed Whitehead's recommendations ${ }^{15}$ for evaluating all interventions aimed at promoting health for their differential impact on socioeconomic groups, and our results could contribute to the development of new overweight/obesity prevention policies. Second, adolescents' SES was measured with the IPSE, an index that is not widely used but allows for taking several dimensions (social, economic, and cultural) of the SES into account. Moreover, there is no perfect indicator to measure SES. Indeed, the latter is frequently measured with three noninterchangeable objective indicators (education, occupation, and income) in research. However, education, occupation, and income capture distinct dimensions of SES ${ }^{22}$ that are differentially associated with outcomes, and their use to study the same phenomena may yield different conclusions. ${ }^{32}$ For example, an inverse relation was observed between SES and weight status in $37.5 \%$ of studies that used family income, in $65.8 \%$ studies that used the father's and/or mother's education, and in $41.7 \%$ that used the father's and/or mother's occupation. ${ }^{27}$ Furthermore, parental education, occupation, and income are not considered the most relevant indicators to measure adolescents' SES. ${ }^{33}$ In addition, 


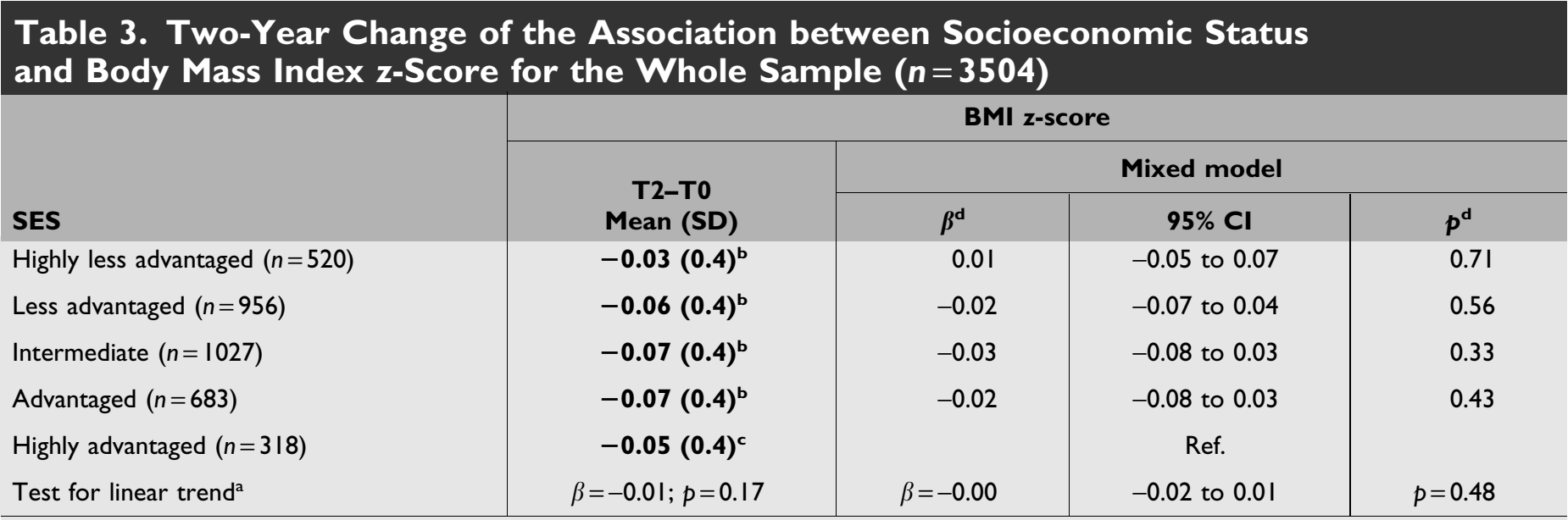

SES defined according to IPSE score ([I-2] highly less advantaged, [3-4] less advantaged, [5-6] intermediate, [7-8] advantaged, and [9-I0] highly advantaged); T0, inclusion; T2, end of intervention. Bold indicates statistical significance.

aIPSE used as discrete variable in each model. The significance $(p)$ of the linear trend test evidenced the presence of a WSG and the slope $(\beta)$ indicated its magnitude.

${ }^{\mathrm{b}} \mathrm{p}<0.000 \mathrm{l}$.

${ }^{c} p<0.05$.

IIPSE used as categorical variable. $\beta$ regression coefficient and associated $p$-value of the mixed model indicated the BMI change of adolescents from highly less advantaged, less advantaged, intermediate, and advantaged IPSE classes compared with those from highly advantaged IPSE class.

$95 \% \mathrm{Cl}, 95 \%$ confidence interval.

\begin{tabular}{|c|c|c|c|c|}
\hline \multirow[b]{3}{*}{ SES } & \multicolumn{4}{|c|}{ Overweight/obesity prevalence } \\
\hline & \multirow{2}{*}{$\begin{array}{c}\text { T2-T0 } \\
\%\end{array}$} & \multicolumn{3}{|c|}{ Mixed model } \\
\hline & & $\beta^{\mathbf{d}}$ & $95 \% \mathrm{Cl}$ & $p^{d}$ \\
\hline Highly less advantaged $(n=520)$ & -0.53 & -0.26 & -0.59 to 0.08 & 0.13 \\
\hline Less advantaged $(n=956)$ & -0.29 & -0.25 & -0.57 to 0.06 & 0.11 \\
\hline Intermediate $(n=1027)$ & $-2.14^{c}$ & -0.40 & -0.72 to -0.08 & $0.0 I^{\mathrm{b}}$ \\
\hline Advantaged $(n=683)$ & -1.90 & -0.40 & -0.73 to -0.07 & $0.02^{\mathrm{b}}$ \\
\hline Highly advantaged $(n=3 \mid 8)$ & 2.20 & & Ref. & \\
\hline Test for linear trend ${ }^{a}$ & $\beta=0.02 ; p=0.97$ & $\beta=-0.01$ & -0.07 to 0.05 & $p=0.73$ \\
\hline
\end{tabular}

SES defined according to IPSE score ([I-2] highly less advantaged, [3-4] less advantaged, [5-6] intermediate, [7-8] advantaged, and [9-I0] highly advantaged); T0, inclusion; T2, end of intervention. Bold indicates statistical signficance.

aPSE used as discrete variable in each model. The significance $(p)$ of the linear trend test evidenced the presence of a WSG and the slope $(\beta)$ indicated its magnitude.

${ }^{\mathrm{b}} \mathrm{p}<0.00 \mathrm{I}$.

${ }^{c} p<0.05$.

IIPSE used as categorical variable. $\beta$ regression coefficient and associated $p$-value of the mixed model indicated the BMI change of adolescents from highly less advantaged, less advantaged, intermediate, and advantaged IPSE classes compared with those from highly advantaged IPSE class. 
the methodology used to create the IPSE is similar to the construction of the standard international socioeconomic index of occupational status, which is a widely used index. ${ }^{24}$ Finally, because the classification of the IPSE scores into five relevant classes was not performed before our study, there was no existing literature to justify how social classes should be created from IPSE. However, the method used was based on previously published works on the subject of social inequalities in health that used an a priori classification of SSE indicators close to IPSE (e.g.: the Family Affluence Scale). ${ }^{22,31}$ Despite these limitations, our study has several strengths. First, our results are derived from data of a trial with a long follow-up and a large sample size. Second, analyses were conducted for the whole sample and by strategies.

\section{Conclusions}

This 2-year follow-up study could not provide evidence that a universal school-based intervention was an effective way to reduce socioeconomic inequalities in weight status among adolescents. These results suggest that interventions with a different approach to this study should be considered to tackle these inequalities. Specifically, interventions should have a universal and proportionate approach that accounts for social differences.

\section{Acknowledgments}

In the name of the PRALIMAP trial group, the authors thank all those who, through their various roles, led to the implementation of this project. The PRALIMAP Trial Group: N. Agrinier, N. Angel, R. Ancellin, E. Aptel, F. Bailly, L. Barthelemy, D. Bezaz, E. Bonsergent, S. Briançon, J. F. Collin, R. De Lavenne, E. Dietz, P. Enrietto, E. Favre, M. Gentieu, E. Gouault, M. Helfenstein, S. Hercberg, F. Kurtz, J. Langlois, P. Laure, E. Lecomte, K. Legrand, E. Lecomte, J. Lighezzolo, P. Marx, A. Y. Omorou, A. Osbery, M. O. Piquee, P. Renaudin, G. Robert, A. Schichtel, S. Tessier, A. Vuillemin, E. Villemin, and M. Wuillaume.

\section{Funding Information}

This research did not receive any specific funding from agencies/institutions in the public, commercial, or not-forprofit sectors.

\section{Author Disclosure Statement}

No competing financial interests exist.

\section{Supplementary Material}

Supplementary Figure S1

Supplementary Table S1

Supplementary Table S2

Supplementary Table S3

Supplementary Table S4

\section{References}

1. Ng M, Fleming T, Robinson M, Thomson B, et al. Global, regional, and national prevalence of overweight and obesity in children and adults during 1980-2013: A systematic analysis for the Global Burden of Disease Study 2013. Lancet 2014;384:766781.

2. Verdot C, Torres M, Salanave B, Deschamps V. Corpulence of children and adults in metropolitan France in 2015. Results of the Esteban study and change since 2006. Bull Epidémiol Hebd 2017; 13:234-241.

3. Gurnani M, Birken C, Hamilton J. Childhood obesity: Causes, consequences, and management. Pediatr Clin North Am 2015;62: 821-840.

4. Singh AS, Mulder C, Twisk JWR, et al. Tracking of childhood overweight into adulthood: A systematic review of the literature. Obes Rev 2008;9:474-488.

5. Sawyer SM, Afifi RA, Bearinger LH, et al. Adolescence: A foundation for future health. Lancet Lond Engl 2012;379:1630-1640.

6. Dinsa GD, Goryakin Y, Fumagalli E, Suhrcke M. Obesity and socioeconomic status in developing countries: A systematic review. Obes Rev 2012;13:1067.

7. Devaux M, Sassi F. Social inequalities in obesity and overweight in 11 OECD countries. Eur J Public Health 2013;23:464-469.

8. Whitehead M, Dahlgren G. Concepts and principles for tackling social inequities in health: Levelling Up Part 1. Copenhagen: WHO Regional Office for Europe. 2007;1-45.

9. Drewnowski A. Obesity, diets, and social inequalities. Nutr Rev 2009;67:S36-S39.

10. Frederick $\mathrm{CB}$, Snellman K, Putnam RD. Increasing socioeconomic disparities in adolescent obesity. Proc Natl Acad Sci U S A 2014; 111:1338-1342.

11. Inchley J, Currie D, Jewell J, et al. Adolescent Obesity and Related Behaviours: Trends and Inequalities in the WHO European Region, 2002-2014. Observations from the Health Behaviour in School-Aged Children (HBSC) WHO Collaborative Cross-National Study. WHO Regional Office for Europe: Copenhagen, 2017.

12. Chung A, Backholer K, Wong E, et al. Trends in child and adolescent obesity prevalence in economically advanced countries according to socioeconomic position: A systematic review. Obes Rev 2016;17:276-295.

13. Haynos A, O'Donohue W. Universal childhood and adolescent obesity prevention programs: Review and critical analysis. Clin Psychol Rev 2012;32:383-399.

14. Moore GF, Littlecott HJ, Turley R, et al. Socioeconomic gradients in the effects of universal school-based health behaviour interventions: A systematic review of intervention studies. BMC Public Health 2015;15:907.

15. Whitehead M. A typology of actions to tackle social inequalities in health. J Epidemiol Community Health 2007;61:473-478.

16. Bambra CL, Hillier FC, Cairns J-M, et al. How Effective Are Interventions at Reducing Socioeconomic Inequalities in Obesity among Children and Adults? Two Systematic Reviews. NIHR Journals Library: Southampton, UK, 2015. DOI:10.3310/phr03010.

17. Briançon S, Bonsergent E, Agrinier N, et al. PRALIMAP: Study protocol for a high school-based, factorial cluster randomised interventional trial of three overweight and obesity prevention strategies. Trials 2010;11:119.

18. Bonsergent E, Agrinier N, Thilly N, et al. Overweight and obesity prevention for adolescents: A cluster randomized controlled trial in a school setting. Am J Prev Med 2013;44:30-39. 
19. de Onis M, Onyango AW, Borghi E, et al. Development of a WHO growth reference for school-aged children and adolescents. Bull World Health Organ 2007;85:660-667.

20. Cole TJ, Bellizzi MC, Flegal KM, Dietz WH. Establishing a standard definition for child overweight and obesity worldwide: International survey. BMJ 2000;320:1240-1243.

21. Insee-Definitions, methods, and quality-PCS 2003-Level 1List of aggregated socio-professional categories. Available at https://www.insee.fr/fr/information/2016811 (Last accessed May 23, 2018).

22. Akkoyun-Farinez J, Omorou AY, Langlois J, et al. Measuring adolescents' weight socioeconomic gradient using parental socioeconomic position. Eur J Public Health 2018;28:1097-1102.

23. Rocher T. Construction of a social position index for students. Educ Form 2016;90:5-27.

24. Ganzeboom HBG, De Graaf PM, Treiman DJ. A standard international socio-economic index of occupational status. Soc Sci Res 1992;21:1-56.

25. Montgomery AA, Peters TJ, Little P. Design, analysis and presentation of factorial randomised controlled trials. BMC Med Res Methodol 2003;3:26.

26. Goodman E, Slap GB, Huang B. The public health impact of socioeconomic status on adolescent depression and obesity. $\mathrm{Am} \mathrm{J}$ Public Health 2003;93:1844-1850.

27. Barriuso L, Miqueleiz E, Albaladejo R, et al. Socioeconomic position and childhood-adolescent weight status in rich countries: A systematic review, 1990-2013. BMC Pediatr 2015;15:129.

28. Hillier-Brown FC, Bambra CL, Cairns J-M, et al. A systematic review of the effectiveness of individual, community and societal level interventions at reducing socioeconomic inequalities in obesity amongst children. BMC Public Health 2014;14:834.
29. Simon C, Schweitzer B, Oujaa M, et al. Successful overweight prevention in adolescents by increasing physical activity: A 4-year randomized controlled intervention. Int J Obes 2008;32:1489.

30. Marmot M. Fair Society, Healthy Lives: A Strategic Review of Health Inequalities in England Post-2010. London: Marmot Review; 2010. Available at www.instituteofhealthequity.org/resourcesreports/fair-society-healthy-lives-the-marmot-review (Last accessed February 6, 2019).

31. Legrand K, Lecomte E, Langlois $\mathrm{J}$, et al. Reducing social inequalities in access to overweight and obesity care management for adolescents: The PRALIMAP-INÈS trial protocol and inclusion data analysis. Contemp Clin Trials Commun 2017;7:141-157.

32. Diemer MA, Mistry RS, Wadsworth ME, et al. Best practices in conceptualizing and measuring social class in psychological research. Anal Soc Issues Public Policy 2013;13:77-113.

33. Koivusilta L, Rimpelä A, Kautiainen S. Health inequality in adolescence. Does stratification occur by familial social background, family affluence, or personal social position? BMC Public Health 2006;6:110.

\section{Address correspondence to: Florian Manneville, $\mathrm{MPH}$ APEMAC, Team MICS University of Lorraine 9 avenue de la Forêt de Haye Vandoeuvre-lès-Nancy Nancy 54505 France} E-mail: florian.manneville@univ-lorraine.fr 